\title{
History of healthcare technology assessment in Japan
}

\author{
Akinori Hisashige \\ Institute of Healthcare Technology Assessment
}

There has been a rapid growth of healthcare technology assessment (HTA) activities among health service researchers and physicians in Japan in the younger generation since the mid-1980s. HTA has become visible since the Ministry of Health, Labor, and Welfare (MHLW) set up the several committees related to HTA in the late 1990s. The MHLW had to participate in regulatory and administrative reform, coping with the serious economic stagnation since 1991, following the economic recession in the 1980s. However, HTA has not been developed as expected. The most important failure is that the application of HTA to health policy has been neglected by the MHLW. Only application to clinical practice has been implemented by developing evidence-based clinical practice guidelines. The MHLW had the main aim of containing costs by reducing excess or useless healthcare services through guidelines, rather than to implement a radical reform. Without a central organization for HTA, several researchers have still continued to do HTA studies, but most researchers and physicians promoting HTA have been moved into diverse related areas. Ultimately, increasing efficiency may be the only way of reconciling rising demands for health care with public financing constraints. Therefore, the reconsideration and reorganization of HTA, which covers not only healthcare services but also the healthcare system as a whole, is becoming an urgent matter for healthcare reform.

Keywords: Health technology assessment, History, Japan, Health policy, Bureaucrat-cabinet system

\section{GENERAL OVERVIEW OF JAPAN}

Japan, lying at the end of East Asia, consists of four main islands and more than a thousand smaller other islands. Its overall area is approximately $370,000 \mathrm{~km}^{2}$. Japan is separated from the Asian mainland by $200 \mathrm{~km}$ of water. The separation from the mainland helped Japan avoid invasion or large-scale immigration from the mainland, while enabling it at the same time to maintain cultural, technical, and intellectual contact with the mainland $(19 ; 24)$. The result is the hybrid culture of Japan, which incorporated cultures from other countries and transformed them into a unique Japanese style $(19 ; 24)$. The population was 128 million in 2006, which is the second largest among Organisation for Economic Co-operation in Development (OECD) countries. It has been declining since 2006 , for the first time in history. As to ethnic groups, more than 99 percent is Japanese and 0.6 percent is other, mostly
Korean. The language is Japanese. Japan is essentially an industrial and trading nation.

The government of Japan is a parliamentary representative democratic monarchy, where the Prime Minister is the head of government. There are multiple political parties. Whereas executive power is exercised by the government, legislative power is vested in both the government and the two chambers of parliament; the Diet with its House of Representatives and its House of Councilors. The Judiciary is independent from the executive and the legislature. Japan is generally considered a constitutional monarchy, based largely upon the British system with strong influences from European continental civil law countries such as Germany. Due to the traditional bureaucracy with a strong power in policy making and its implementation, the Japanese governance is essentially a bureaucrat-cabinet system, rather than a parliament-cabinet system (16). The Liberal Democratic 
Party has been in power since 1955, except for a short-lived coalition government in 1993.

Whereas gross domestic product (GDP) is an indicator of the size of country's economy, GDP per capita is a broad indicator of the prosperity of its population. GDP per capita in Japan is $\$ 30,776$ in 2005 . GDP is indicated as a dominant determinant of healthcare expenditure, contributing over 90 percent to its variance, through many cross-national and longitudinal studies (3). In 2005, OECD countries spent an average 9.0 percent of their GDP on health care, up by 0.9 percent from 2000. However, healthcare expenditure in Japan accounted for 8.0 percent of GDP, one percent lower than the average. Japan also ranks below the OECD average in terms of health spending per capita, compared with an OECD average of $\$ 2,759$ in 2005 . The growth rate in health spending in Japan is also lower than the OECD average of 4.3 percent.

As to health status, Japan enjoyed the highest life expectancy among OECD countries, with 82 years for the population as a whole. The healthy life expectancy, which combines life-expectancy and quality of life, is the longest in Japan, closely followed by European countries (20). Infant mortality rates in Japan is the lowest, roughly half the OECD average. From a macro economics perspective, healthcare efficiency could be estimated by comparing health expenditure to life expectancy. The cost-effectiveness ratio in Japan is relatively low among developed countries. This suggests that Japan's healthcare system has attained high-performance.

\section{THE HEALTHCARE SYSTEM IN JAPAN}

The healthcare system in Japan is a mixture of centralized governance such as seen in the United Kingdom, social insurance financing as in Germany, and private healthcare provision as in the United States. That is to say, it is a hybrid system of Beveridge, Bismarck, and market models (14). The characteristics of the healthcare system are summarized as follows $(4 ; 6 ; 8 ; 14)$. Health care in Japan is highly controlled and regulated by the Ministry of Health, Labor, and Welfare (MHLW). The MHLW covers a wide range of activities: health policy development, health information management, monitoring of health status and performance of health sectors, and regulation of social insurance funds. In 2004, 85 percent of revenue came from public funds.

The Japanese health insurance system has consistently expanded its coverage and benefits, and it has universally covered all citizens, since $1961(4 ; 6 ; 8 ; 14)$. However, there is neither the consumer's choice of health insurers nor the insurer's choice of service providers. The health insurance system is complex and fragmented. The present system consists of three main schemes, with diverse variations among them: the employees' health insurance, the self-employees' and pensioners' health insurance, and the Geriatric Health Act.
The reimbursement system is rather simple $(4 ; 6 ; 8 ; 14)$. The nationally uniform fee schedule lists all procedures and products, including drugs, which can be reimbursed by health insurance. The same fee is paid to all providers for the same procedures and products, without consideration of costs. There is no differentiation between physician fee and hospital fee in the schedule. Payments for outpatient care are predominantly on a fee for service basis. Inpatient care is paid through a mixture of per diem and fee for service. The fee schedule, which is revised every 2 years by the government, plays a key role in the distribution of healthcare resources in Japan. All revenues of healthcare providers and the government budget for subsidies are highly dependent on the fee schedule.

The characteristics of health care in Japan are summarized as follows $(4 ; 6 ; 8 ; 14)$. The first characteristic is private sector dominance. The majority of hospitals are owned and run by individual physicians. The remaining hospitals, mostly large, are public sector or nongovernment organizations. For profit, investor-owned hospitals are prohibited. Japanese physicians are private sole general practitioners in clinics, essentially providing primary care. Specialists mainly work in hospitals on salary. Practitioners cannot attend to hospitalized patients, and hospital physicians are not allowed to have a private practice on the side.

The second characteristic is drug overutilization. As a component of healthcare expenditure, drugs are still in an important position. Japanese physicians not only prescribe but also dispense drugs. They usually purchase drugs at discounted prices. Approximately half of all prescriptions are dispensed by physicians. The third characteristic is high accessibility. The unrestricted access to even tertiary hospitals results in no waiting lists for treatment. Because provision is weighted toward outpatient treatment, Japan has one of the highest rates of physician visits and the lowest rate of hospital admissions among developed countries. The fourth characteristic is low staffing. The number of practicing physicians per 1,000 population in Japan is well below the OECD average. The number of nurses per bed is extremely low.

The fifth characteristic is discontinuous services. There is no functional differentiation between acute and long-term care among hospitals. Many hospital beds are actually functioning for nursing care. The sixth characteristic is healthy life style. Life-style, as well as economic conditions, seems to be the main factors of health improvement in Japan. The prevalence of obesity and diabetes in Japan, or the incidence of breast cancer and myocardial infarction is extremely low compared with other OECD countries. The seventh characteristic is technology preference $(4 ; 6 ; 8 ; 14)$. The number of magnetic resonance imaging (MRI) and computed tomography (CT) units per million population in Japan is extraordinary high. In contrast, invasive therapeutic technology has not much diffused.

The last, but the most important characteristic, is lack of attention to the quality of healthcare services $(4 ; 6 ; 8 ; 14)$. For 
example, from prevention to treatment, only a limited number of randomized controlled trials (RCTs), which are the gold standard for determining effectiveness, have been carried out in Japan. A large number of healthcare technologies have been introduced and used uncritically.

\section{RISE OF HEALTHCARE TECHNOLOGY ASSESSMENT}

The introduction and diffusion of healthcare technologies are determined by a complex interaction of market forces, public funding, and regulation. The main avenue for controlling the introduction and use of healthcare technologies has been a financial mechanism through fee-schedule setting and revision for reimbursement.

The general characteristics of policy decision making in the Japanese government are bureaucracy-dominant and implicit $(5 ; 8 ; 16)$. Health policy is developed and adjusted through consensus among related Ministries and stakeholders such as politicians, industries, clinicians, and experts $(5 ; 16)$. Moreover, the policy decision making is mainly based on experience and intuition with limited evaluation. Under these circumstances, the diffusion and utilization of healthcare technologies have been relatively free, although the fee-schedule is at low level. On the other hand, there are few incentives for critical evaluation of these policies among healthcare providers, because clinical practices are paid on fee-for-service basis.

\section{Technology Assessment}

In Japan, despite escalating healthcare expenditure, the suppression of healthcare expenditure was successfully achieved in comparison with other developed countries by a rigorous cost-containment policy (14). However, economic recessions in the 1970 s caused serious financial crisis for social security schemes, including health insurance. The Japanese government started regulatory and administrative reforms, including healthcare reform. The government implemented comprehensive and thorough cost containment measures: budget ceilings, revision of fee schedules, reduction in drug prices, and so on. An international approach of healthcare policy has been shifted from simple cost-containment strategies to long-term structural change for improving value-for-money in health care. However, this attempt was not led by the Ministry of Health and Welfare, but by other Ministries and Agency.

In the 1960s, Japanese society was faced with serious environmental pollution by industries throughout the country. Also, the transparency and accountability of big public projects for science and technology were seen as problems. At the beginning of the 1970s, technology assessment was officially mentioned in the report or white paper of the government and pointed out to be introduced to cope with problems. Both the Science and Technology Agency (STA) and the Ministry of International Trade and Industry (MITI) investigated technology assessment activities in the United States and carried out more than 60 case studies on technology assessment studies independently.

After the economic recession in 1973 caused by the Oil Shock, industries lost interest in HTA and rather expressed fears about strict regulations through HTA. The Council for Science and Technology attempted to create a law for HTA, but failed. Although HTA projects were continued at the beginning of the 1980s, they never went beyond the pilot stage. Only environment assessment has been established as part of a formal legislative framework. This rise and fall of HTA in Japan is noteworthy as a typical example of a building an explicit and comprehensive framework into policy decision making. Unfortunately, it had no impact or influence on health care and its policy.

\section{Healthcare Technology Assessment}

While the Ministry of Health and Welfare (MHW) was muddling through the serious crisis of health care during the late 1970s and the early 1980s, without any strategies for drastic reform, several physicians and healthcare researchers in the younger generation, who studied in the United States around the mid-1980s, tried to cope with this critical situation and intended to change it (10). They obtained training for new disciplines (e.g., healthcare technology assessment [HTA], clinical epidemiology, clinical economics, and medical decision making) at the Harvard School of Public Health and/or the Clinical Epidemiology Unit at the University of Pennsylvania, which were the Mecca of these disciplines. At that time, these disciplines were becoming visible internationally.

In 1985, the Japanese Association of Medical Technology Assessment was organized by physicians, healthcare researchers, health economists, medical historians, and bioethicists (10). The constitution of members reflected their aim to change the paradigm of health care. They carried out extensive promotional and educational activities to disseminate HTA and other related areas such as clinical decision analysis, clinical economics, and clinical epidemiology (i.e., evidence-based medicine [EBM]). Thereafter, the Association of Medical Decision Making and the Association of Clinical Economics were organized. Although the number of the association was approximately 150 , the number of core members who were doing research on HTA was very limited.

In 1988, the International Symposium of Medical Technology Assessment was held in Tokyo. The keynote speakers were Drs. Seymour Perry, Harvey Fineberg, and Donald Berwick. Simultaneously, the International Workshop of Medical Decision Making was held. Officials of the MHW, researchers, and journalists have paid increasing attention to issues concerning HTA. However, the actual movement for changing health policy was not observed in the MHW. 


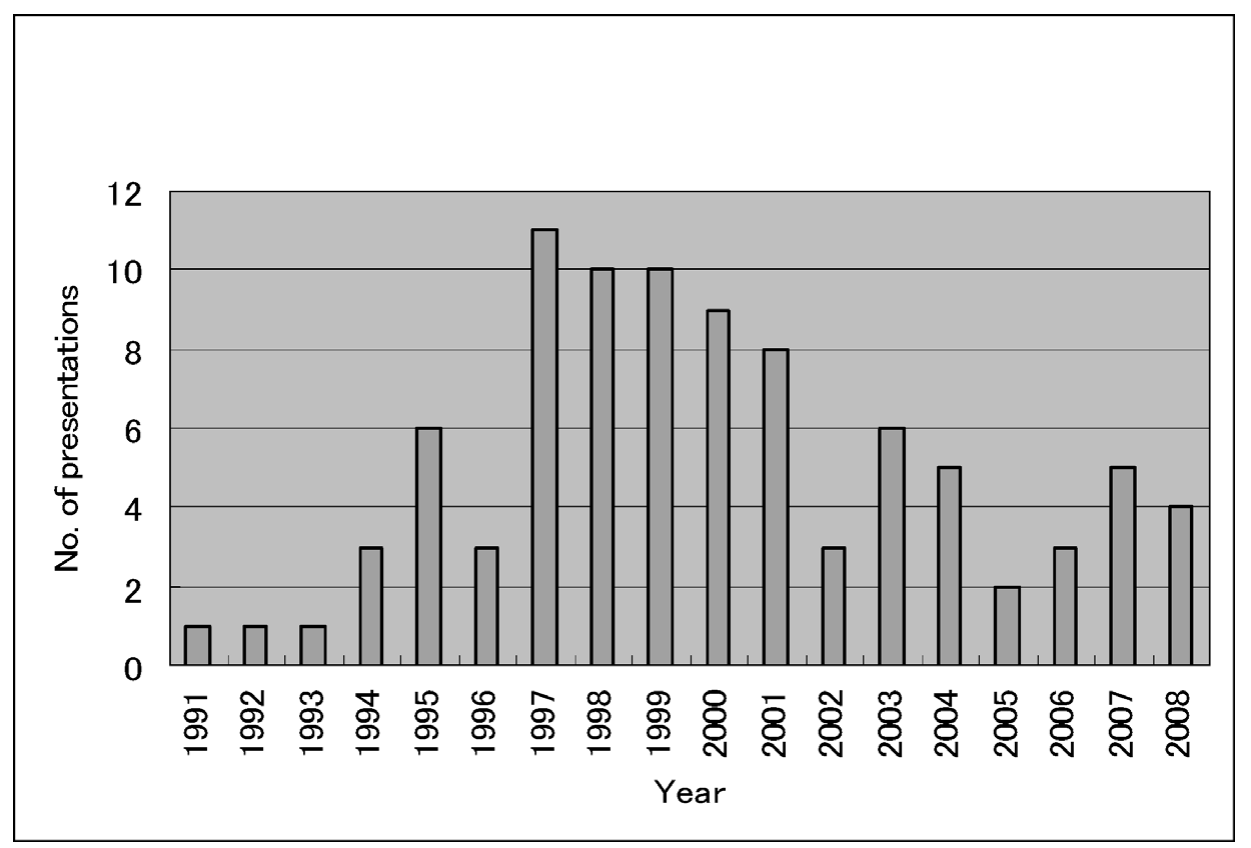

Figure 1. Trend of presentations by Japanese researchers at the International Society of Technology Assessment in Health Care (ISTAHC) and Health Technology Assessment international (HTAi) meetings.

\section{DEVELOPMENT OF HTA}

\section{Research Activities}

The number of researchers on HTA was gradually increasing and activated their works in Japan. Figure 1 shows the trend of presentations by Japanese researchers at the International Society of Technology Assessment in Health Care (ISTAHC) and Health Technology Assessment international meetings (HTAi) since 1991 (18). The number of presentations increased rapidly from 1991 to 1997, and has decreased since then. Because 48 percent of these presentations were presented by Hisashige and his group, the number of HTA researchers in Japan was limited. Table 1 shows the category of presentations. As to themes of the presentations, the proportion of cost-effectiveness analysis was the highest (29 percent), followed by quality of life measurement (15 percent), screening issues (15 percent), and health service utilization (11 percent). The presentations reflected the issues of health care in Japan, where information on economic evaluations or quality of life was greatly lacking. The assessment of screening was timely and important, because diverse nationwide screening programs were introduced without critical evaluation and its evidence.

Although HTA activities in Japan appeared to the outsider as important compared with those of other Asian countries in the early $1990 \mathrm{~s}$, these activities, as well as the Japanese healthcare system and its sociocultural background, were regarded as a black box by the Western world. In 1994, a special issue for HTA in Japan (vol. 10, no. 3, edited by Ikegami and Hisashige) was published in the ISTAHC journal. This issue covered a wide range of HTA in Japan,
Table 1. Themes Presented by Japanese Researchers at the ISTHAC and HTAi Meetings

\begin{tabular}{lc}
\hline Theme & Number (\%) \\
\hline Cost-effectiveness analysis & $26(29)$ \\
Quality of life measurement & $14(15)$ \\
Screening issues & $14(15)$ \\
Health service utilization & $10(11)$ \\
Diagnostic tests & $6(7)$ \\
Preference measurement & $4(4)$ \\
Needs assessment & $4(4)$ \\
Clinical guidelines & $2(2)$ \\
Efficacy of clinical services & $2(2)$ \\
Decision making & $2(2)$ \\
Diffusion of health technology & $2(2)$ \\
Information technology & $2(2)$ \\
Others & $3(3)$ \\
Total & $91(100)$ \\
\hline
\end{tabular}

from screening and diagnostic imaging to cancer treatment and bioethics around brain death.

\section{Government Advisory Committees Promoting HTA}

Following the collapse of the bubble economy in 1991, the Japanese economy stagnated suddenly, and this stagnation has continued (14). Healthcare expenditure has outpaced economic growth over the past decade. The budget ceiling for social security was set at a level of 0 percent increase. Even worse, the Japanese government has been running a large fiscal deficit, more than 6 percent of GDP. Whereas cost 
Table 2. Report of the Advisory Committee of the Application of Healthcare Technology Assessment, 1997

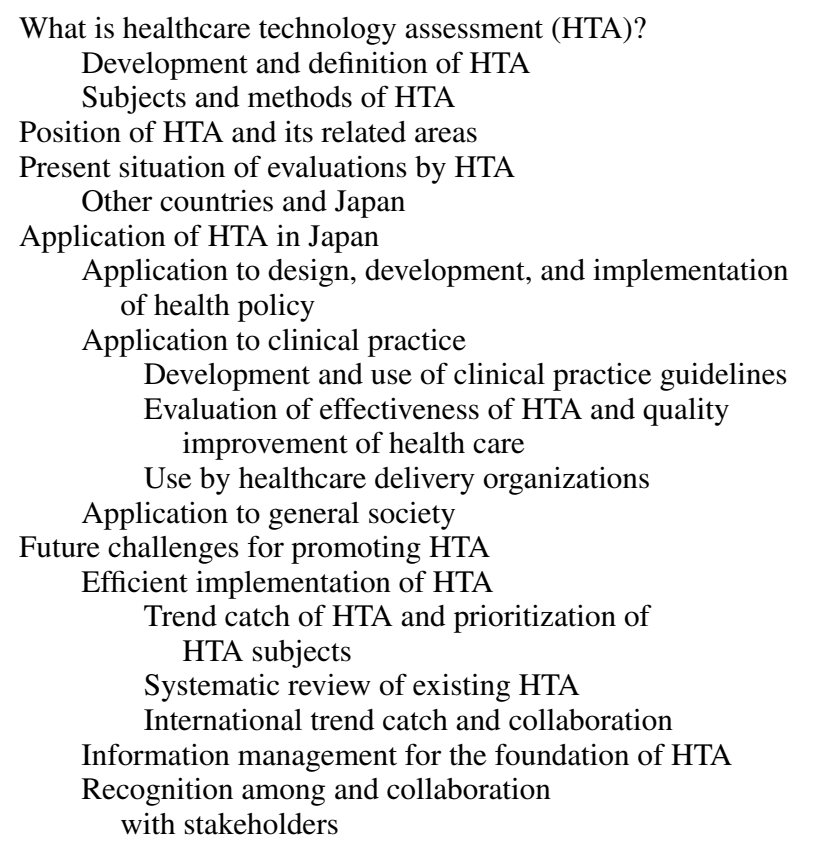

containment became more rigorous than before, efficiency and quality assurance of health care were needed. Although the Japanese government has not had an explicit health policy to comprehensively cope with these issues, it has gradually tried to examine new challenges for policy decision making.

In 1996, the MHW organized the Advisory Committee of the Application of HTA (22). Officially, it was the first commitment to HTA by the MHW. The main aim of this committee was the application of HTA to improve quality of health care and provide efficient health care. After discussions and debate, in 1997, the committee issued the report as shown in Table 2. Its contents covered a wide range of issues related to HTA, which reflected the international trends of HTA and concisely summarized characteristics of HTA compared with closely related other disciplines (e.g., EBM, quality improvement, critical path, and so on). It was a nice starting point for introduction and diffusion of HTA in Japan. However, there was neither action plan nor financial support for realizing statements of this report. The most important issue not mentioned was who or what organization was mainly responsible for carrying out or managing these activities.

Just before this report, the White Paper of Health and Welfare (21) was published and introduced HTA in the chapter, entitled "health science and technology assessment," for the first time. It explained the development of health technologies and their assessment with several examples addressed to ordinary people. This was one of future challenges for promoting HTA.

In 1998, the MHW organized the Advisory Committee of Promotion of Health Technology Assessment (22). The aim of this committee was the examination of EBM to implement or apply HTA in clinical practice, recognizing the international rapid acceptance of EBM since 1992. The next year, the report of the committee was issued. The key conclusion was the development of evidence-based clinical practice guidelines (CPGs) to disseminate and promote EBM. After priority setting for CPGs, projects for the development of evidence-based CPGs have been funded by the MHW. The MHW had completed shifted its focus from HTA to EBM.

As to the coordination toward integrated promotion of HTA and EBM, the need for establishing a center, like the Agency for Health Care Policy and Research in the United States or the National Institute of Clinical Excellence in the UK, was pointed out. Also, in 1999, as a part of research on HTA in Japan (the chief investigator, A. Hisashige) funded by the MHW, Drs. Egon Jonsson and David Banta visited Japan and wrote reports giving recommendations for promoting HTA in Japan. These reports recommended that a mature next step in HTA development would be to establish a national mechanism, a co-coordinating center or a council, which could ensure a sustainable and systematic production of evidence about effective and cost-effective health practices in Japan. They also suggested that such a national mechanism for HTA should serve as a link between research and policy making. However, the government has not established the formal organization of HTA and EBM.

Following a statement about an information network for EBM promotion in the former report, in 2001, the Ministry of Health, Labor and Welfare (MHLW) organized the Advisory Committee of Support for Dissemination of Information on Healthcare Technology. The basic aim was to discuss and develop a plan to supply or support information useful for healthcare professionals and patients at clinical settings. A comprehensive plan (11) was proposed and discussed to establish a national health information center, which could cover a wide range of information from knowledge and patient information to know how and knowledge management, like the National Electronic Library for Health in the United Kingdom. Finally, due to a lack of financial support, a plan was narrowly specified as a guideline information database. The Medical Association was strongly against a plan to build an information center in organizations related to the MHLW. In 2004, the Medical Information Network Distribution Service was set up at the Web site of the Japan Council for Quality Health Care, which was established by the collaboration between the government and the association in 1985.

\section{PRIORITY SETTING, FUNDING, AND EVALUATION OF HTA}

The core funding for research on HTA had been the national funds for health and welfare of the MHW (now, the MHLW). Whereas sometimes, researchers or physicians carried out HTA, pharmaceutical companies and medical device 
industries seldom supported HTA. For health insurance application, they voluntarily submitted economic evaluations of their products, but these evaluations were far from HTA and mostly poor in quality. The funding for HTA by the MHW started in 1990 just after the international symposium of HTA in Japan. The research themes were diagnostic imaging, diagnostic tests, and medical technologies. The next year, it was expanded from diagnostic technology and treatment technology to preventive and information technology. Since then, this funding continued but fluctuated in amounts.

The individual technologies assessed were MRI, CT, gene therapy, low-invasive treatment, organ transplantation, and so on. After the advisory committees on HTA, in 1999, as a strategy of research and development for health science, a top priority was put on EBM and HTA. In 2000, the total fund for the Research for Science of Health and Welfare was approximately $\$ 290$ million, and that for the Comprehensive Assessment of Medical Technologies, which was related to HTA or EBM, was approximately $\$ 10$ million (17). Whereas the fund for genuine HTA was $\$ 0.2$ million, those for guidelines and EBM were $\$ 1.8$ million and $\$ 1.2$ million, respectively. This reflected the shift of the health policy of the MHLW, although HTA for health policy had been indicated as the basis for HTA development. Also, the MHLW generally tends to use only certain results of HTA research rather than them as a whole, depending on interest in the NHLW.

In implementing evidence-based strategies, it is essential to identify needs for HTA and set their priority, because the infrastructure for HTA, which requires a long-term investment, has been greatly lacking in Japan, and HTA itself needs considerable resources. The MHLW has officially done neither a needs assessment nor priority setting. Hisashige carried out a survey for needs assessment and priority setting for HTA in HTA research funded by the MHW (9). The subjects of the survey were 1063 random samples from councilors or board members of sixteen major medical associations. High-ranking priority scores of healthcare technologies among candidates, which were cited by more than ten people and expected to have an impact within 5 years, are rehabilitation (12.7), healthcare system issues (12.6), cancer screening (12.3), cancer chemotherapy (12.2), counseling for life style (12.1), home care (12.1), CT for cancer (11.9), PTCA (11.7), artificial joints (11.7), minimally invasive therapy (11.6), and so on. This was the first priority assessment on HTA. Although the importance of priority setting was indicated in the report of the committee, the MHW has not tried to explicitly explore the priority of HTA.

At the same time, no explicit effort has been made to evaluate benefits or payback of health policy researches by the MHLW, before and/or after their funding, although there has been an implicit system for before and after assessment of these studies. The committees of experts selected by the MHW have assessed the usefulness, sometimes using a scoring tool. One survey (12) examined the issues of assessment in healthcare research funded by the MHW. Among twentytwo health policy research projects, the proportion of an item with any description was highest in utilization (45.5 percent) and lowest in the cost of illness and the acceptance of new knowledge among doctors (4.5 percent). In general, the proportion in items for clinical effects was relatively higher than that for quality and economic issues, although the overall proportion was extremely low. This result shows that their quality was insufficient to inform the health policy decision makers about priority settings in funding them.

To promote evidence-based health care, a system, which ensures the benefit or payback from research, should be established in the formal function of the MHW. Moreover, appropriate guidelines or guidance should be developed and recommended to researchers to promote a dialogue between policy makers and researchers. Since 2003, the MHLW has introduced an assessment system for researchers funded by the Research for Science of Health, Labor and Welfare, before and after their implementation. The method is a qualitative and descriptive evaluation about three items (i.e., necessity, efficiency, and effectiveness). It seems to be transparent compared with the former method, but poor in quality and far from an explicit and multidimensional assessment.

\section{PRESENT SITUATION}

\section{Evidence and Guidelines}

In Japan, evaluation of effectiveness and efficiency of health care has been greatly lacking, as mentioned before (8). This has hampered the development of HTA or EBM in Japan, and a large number of healthcare technologies have been diffused and used without critical evaluations. RCTs, which are the gold standard for determining effectiveness, have seldom been carried out in Japan. In the last decade, the degree of Japan's contribution to the articles in major international journals was only 0.7 percent in clinical research, which was much lower than that in basic (3.1 percent) research (2). This difference seems to be due to the medical tradition introduced from Germany in the Meiji era (6).

Pharmaceuticals are the only exception evaluated by RCTs under the strict regulation. However, the quality of RCTs had been relatively poor $(8 ; 25)$, and many drug-induced disasters occurred. Since 1997, the new and strict guidelines of the International Conference on Harmonization-Good Clinical Practice have been introduced and effective (23). They are considered to improve the quality of RCTs. However, the recent study based on the good clinical practice audits showed a decrease in the proportion of case report form-related deficiencies, but an increase in the proportion of protocol deviations.

The deficiency of high-quality clinical evidence has influenced development of evidence-based guidelines in Japan compared with recent international EBM developments. The quality scores of typical existing CPGs in Japan were 
extremely low in comparison with those of the national UK CPGs, as well as international CPGs (13). This is more or less true among twenty-three evidence-based guidelines funded by the MHLW. Although a large part of evidence used in developing guidelines have been created in the United States and Europe, there should be methodological development for translating them into the Japanese context. Therefore, recent evidence-based guidelines in Japan have a face value of following the standard format, but their essential part has serious problems.

Moreover, there are several controversial issues in evidence-based guidelines in Japan. First, the development of most evidence-based guidelines, mainly funded by the MHLW, was heavily delegated to experts in each academic association. Specialists in HTA and EBM did not play an important role and stakeholders such as patients or primary care physicians were not or seldom involved. Second, there is very limited information about conflicts of interest. Internationally, it is pointed out that 87 percent of CPG authors had some form of interaction with pharmaceutical industry (1). In Japan, this interaction should be taken more seriously, because academic researchers have been strongly dominated by the feudalistic hierarchy (6) and their leaders have been closely related to pharmaceutical development. Even in a preventive area, most authors of guidelines or systematic reviews have been closely related to or promoting screening programs in local areas.

\section{Pharmaceuticals}

Pharmaceuticals are the most important health technology in Japan fiscally. The expenditure was $\$ 73$ billion in 2005, which is the second largest market of drugs in the world. Overutilization of drugs is one of the issues in health care in Japan $(8 ; 14)$. The MHLW and other government pharmaceutical regulatory organizations have implemented major changes to strengthen their review of drugs. In 2004, integrating existing related organizations, the Pharmaceutical and Medical Devices Agency (PMDA) was established. The role of the PMDA is to provide consultations concerning the clinical trials of new drugs and medical devices, and to conduct approval reviews and surveys of the reliability of application data. Following this reorganization, the MHLW and PMDA handle a wide range of activities from clinical studies to approval reviews, postmarketing surveillance, and safety measures. The goal is to improve the safety and quality of drugs entering the Japanese market.

The Japanese pharmaceutical industry has developed and marketed marginally innovative dugs $(8 ; 14)$. The pricing formula for new pharmaceuticals in Japan is essentially based on a comparison with the price of a similar drug on the market. Then, price was set at a high level for even noninnovative drugs. However, the fee-schedule for drugs has been revised every 2 years by reflecting market (i.e., purchase) price. The market price has continuously decreased, because healthcare providers and dispensers purchase drugs at discount rates. However, together with policy decisions, the fee-schedule of drugs has continuously been declining at the rate of 5 percent to 10 percent. The increase of average fee-schedule of all healthcare services has been compensated by drugs fee-schedule cutting.

Economic evaluation of health care has not been active in Japan, because there is no regulation requiring economic evaluation for health care by the government. The MHLW has not made any regulation reform for considering costeffectiveness or value for money of drugs. The pricing formula for new drugs does not reflect drug quality in terms of efficacy, effectiveness and/or cost. In 1992, the presentation of information about economic evaluation of drugs as reference materials was suggested by the MHW. However, there was neither explicit influence on pricing decisions nor formal guidance, although a summary sheet for economic evaluation was presented in 1994. Because the quality of materials submitted varied widely and the MHW did not consider them seriously, pharmaceutical companies gradually lost their incentives. The Subcommittee of Basic Problems of Reimbursement has discussed the future pricing formula and put cost-effectiveness at the end of a discussion list, but actually never examined its introduction. This regulation with poor quality of drugs evaluation has promoted the diffusion of less-effective and less-efficient drugs in Japan.

\section{Medical Devices}

Total sale of medical devices in 2005 was $\$ 2.6$ billion, which is approximately one-third of that of drugs. The main components of the market are diagnostic imaging systems (23 percent), surgical instruments, and supplies (16 percent), biofunction substitute/assistive devices and artificial organs (11 percent), and biophenomena monitoring/testing devices (11 percent). The Japanese market is the second largest in the world. Japan imports approximately 40 percent of its medical devices from other countries, mainly from the United States.

The regulatory system of medical devices in Japan has come under widespread criticism for its excessive delays, complicated requirements, nontransparency, insufficient number of qualified reviewers, and expense in gaining product approvals. Since the beginning of the 2000s, legislative systems have been significantly restructured. The assessment method for effectiveness and safety for medical devices become very similar to drugs. For example, clinical trials are required for devices with high risk. However, because the pricing formula is essentially based on the same as drugs, the MHLW does not have an explicit policy for cost-effectiveness of medical devices.

Under the strong and urgent pressure on healthcare expenditure, the price difference of medical devices between Japan and other countries (particularly the United States) has been noted. The market prices of medical devices (i.e., PTCA 
catheters and cardiac pacemakers) in Japan were two to four times higher than those in the United States, whereas those for MRI, CT, and laparoscopies were at the same level. In 2002, the MHLW introduced the foreign price adjustment rule to reduce reimbursement prices in Japan. However, the price difference has not sufficiently been resolved (15).

As to introduction and distribution of big-ticket medicals devices such as MRI or CT, the MHLW has no explicit policy, and healthcare providers and the medical device industry have been left relatively free (7). Japan has an incredibly large number of CT and MRI devices. The MHLW is trying to control their diffusion only through fee-schedule and does not have any explicit plan for regional distribution. Moreover, the utilization of these devices in Japan is relatively low compared with that in the United States. This inefficient diffusion and utilization of medical devices should be eliminated by establishing a comprehensive HTA framework from introduction to diffusion.

\section{FUTURE CHALLENGES}

As examined above, HTA has now been implemented in Japan for more than 25 years. Even though there has been a rapid growth of HTA activities, HTA has not been developed as much as expected. HTA was becoming visible since the MHLW consecutively set up the committees related to HTA in the late 1990s. However, the application of HTA to health policy has been neglected by the MHLW, despite the recommendations of the HTA committee.

There are several possible reasons why the MHLW did not develop the strategy adopting HTA. First, comprehensive evaluation through HTA would make clear the failure of health policy previously implemented. The bureaucrats wish to maintain the myth of infallibility and therefore cannot admit their faults. An independent evaluation from outside has the danger of making these faults clear. Second, the MHLW still relied on the traditional consensus method based on opinion or experience, which favors incremental and marginal changes, rather than a drastic reform. Third, there has been a power game of politics between innovative and conservative groups in the MHLW. In addition, the implementation of specific health policy sometimes depends on the idea of an individual leader, and this may make it somewhat temporary.

The MHLW has been the key player taking the initiative in healthcare policy, because the healthcare system in Japan is highly centralized and has been mainly controlled by its bureaucracy, under the monopoly of the Liberal Democratic Party. Observing the attitudes of the MHLW staff mentioned above, medical professional societies, health insurance organizations, and hospitals did not move actively into HTA. These organizations have also relied heavily on implicit consensus development and are concerned that HTA may be a considerable challenge to this traditional method. In particular, the Japanese Medical Association is reluctant to introduce HTA or EBM, because it represents general practitioners in clinics, who represent the older generation and practice traditional opinion-based medicine.

The desirable course would be to develop an independent HTA agency or establish a division of HTA within the MHLW. Obviously, this has not happened. Researchers and physicians promoting HTA have been scattered over diverse related areas such as EBM, healthcare management, clinical economics, quality assurance, health service researches, and so on. Several key figures have still continued to do research on HTA, but the Japanese Association of Medical Technology Assessment unfortunately died in the course of time.

Faced with economic stagnation and deflation for more than 10 years, the need for a comprehensive reform of the healthcare and insurance system has been recognized by the government (14). However, the MHLW has continued to follow the traditional cost containment policy, which has undermined the infrastructure of health care for several decades. This is reflected in the frequent occurrence of medical errors and the shortage and geographical imbalance of physicians (in particular obstetricians and pediatricians) and nurses. Also, projects for the introduction of market oriented health care were tried but failed, perhaps because they were not based on evidence concerning possible effects or the sociocultural background.

Japanese health policy will inevitably allow a reasonable increase of healthcare expenditure beyond blunt cost containment (14). There are only limited possibilities for increasing resources for health care, including an increase of the premium rate or a transfer of an increase of consumption tax to health care or social security. Because both cases are political matters, they require acceptance of people based on their value judgments. Spending more is not necessarily a problem, particularly if the added benefits exceed the extra costs. However, under the fiscal crisis situation, there are many competitors for resource allocation of the government budget, such as education, defense, pensions, and public works. It will be needed for healthcare professionals and policy makers to compete for resources and assure quality and efficiency of health care, through healthcare reform and information disclosure.

The efforts to incorporate systematic evaluation of health care and its system from a wider perspective are invaluable for realizing this health policy. Therefore, the reconsideration and reorganization of HTA, which covers not only healthcare services but also the healthcare system as a whole, are becoming an urgent matter for healthcare reform. It is a paradigm shift of health policy and its framework, but valuable to be considered in the context of the Japanese healthcare system.

\section{CONTACT INFORMATION}

Akinori Hisashige, MD. PhD (akih@k3.dion.ne.jp), Director, Institute of Healthcare Technology Assessment, 2-24-10, Shomachi, Tokushima, Japan 770-0044 


\section{REFERENCES}

1. Choundhry NK, Stelfox HT, Destky A. Relationship between authors of clinical practice guidelines and the pharmaceutical industry. JAMA. 2002;287:612-617.

2. Fukui T, Rahman M. Contribution of research in basic and clinical sciences in Japan. Intern Med. 2002;41:626-628.

3. Getzen TE, Poullier JP. International health spending forecasts: concepts and evaluation. Soc Sci Med. 1992;34:1057-1068.

4. Health and Welfare Statistics Association. Trends of national health and welfare. J Health Welfare Stat. 2007;54:1-500 (in Japanese).

5. Hisashige A. Risk assessment/management and education for medical decision making in Japan. Toxicol Ind Health. 1991;7:485-494.

6. Hisashige A. Health care delivery, financing system and aging in Japan. J Jpn Assoc Radiol Technol. 1992;39:27-52. (English issue).

7. Hisashige A. The introduction and evaluation of MRI in Japan. Int J Technol Assess Health Care. 1994;10:392-405.

8. Hisashige A. Healthcare technology assessment and the challenge to pharmacoeconomics in Japan. Pharmacoeconomics. 1997;11:319-333.

9. Hisashige A. Need assessment and priority setting for healthcare technology assessment in Japan. In: Proceedings of the 15th Annual Meeting of ISTAHC, Edinburgh, 1999.

10. Hisashige A. Evidence-based medicine. II. The history of EBM and its role. Aimic. 1999;20;4-8. (in Japanese).

11. Hisashige A. Role and trend of information center related to CPGs. In: Hisashige A, ed. Research on healthcare technology assessment funded by the Ministry of Health. Tokyo: Ministry of Health; 2001;24-41. (in Japanese).

12. Hisashige A. Priority setting of health care research in Japan: a critical appraisal of health policy research quality of health policy researches. In: Proceedings of the 19th Annual Meeting of ISTAHC, Alberta, Canada, 2003.

13. Hisashige A. Assessment of the quality of clinical practice guidelines in Japan. In: Proceedings of the 17th Annual Meeting of ISTAHC, Philadelphia, Pennsylvania, 2001.

14. Hisashige A. Japan. In: Rapoport J, Jacobs P, Jonsson E, eds. Cost containment and efficiency in national health systems. New York: Wiley-Blackwell; 2008.

15. Ide $H$, Yasunaga $H$, Imamura $T$, et al. Price differences between Japan and the US for medical materials and how to reduce them. Health Policy. 2007;82:71-77.

16. Iio J. The structure of governance in Japan. Tokyo: Chuokoronsha; 2008. (in Japanese).

17. Institute of Research on Science of Health and Welfare. Front of science of health and welfare, Tokyo: Institute of Research on Science of Health and Welfare; 2000.

18. International Society of Technology Assessment in Health Care and Health Technology. Assessment international. Abstract books, from 1991 to 2008 .

19. Kato S. The hybrid culture, a small hope of Japan. Tokyo: Kodan-sha; 1956

20. Mathers C, Sadana R, Salomon J, et al. Healthy life expectancy in 191 countries. Lancet. 1999;357:1685-1691.

21. Ministry of Health and Welfare. Health science and technology assessment, the White paper of Health and Welfare. Chapter 5. Toward improvement of health and quality of life. Tokyo: Gyousei; 1997.

22. Research and Development Division, Health Policy Bureau, Ministry of Health and Welfare. Straightforward EBM course. Tokyo: Kouseikagaku-Kennkyusyo; 2000. (in Japanese).

23. Saito K, Kodama Y, Ono S, et al. Quality of Japanese clinical trials estimated from good clinical practice audit findings. Am J Ther. 2006;13:127-133.

24. Umesao T, Tada M, eds. The structure of Japanese culture. Tokyo: Kodansha; 1972. (in Japanese).

25. Yanagi M. Levels of clinical trials and its trends from a international perspective. In: Proceedings of the 1 st Pharmacovigilance Seminor, Osaka, Japan. 1999:342-348. (in Japanese). 